\title{
Are Stone-Wales defect sites always more reactive than perfect sites in the sidewalls of single-wall carbon nanotubes?
}

\author{
Xin Lu, ${ }^{* a}$ Zhongfang Chen, ${ }^{b}$ Paul v. R. Schleyer ${ }^{b}$
}

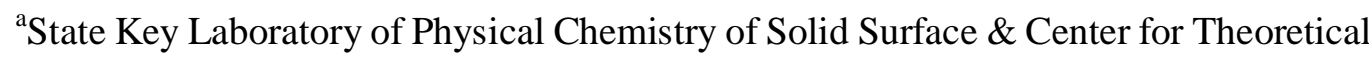
Chemistry, Department of Chemistry, Xiamen University, Xiamen 361005, CHINA;

${ }^{\mathrm{b}}$ Department of Chemistry, University of Georgia, Athens, GA 30602-2525 USA

E-mail: xinlu@xmu.edu.cn

Density function computations using periodic boundary condition (PBC) on the addition of $\mathrm{O}_{3}$ to the sidewall of an infinite $(5,5)$ single-wall carbon nanotube (SWCNT) without and with a Stone-Wales defect were performed using 120-atom supercells for the defect-free and defect tubes (depicted in Fig. S1a and Fig. S2a, respectively).

These PBC computations employed the ultrasoft pseudopotential ${ }^{\mathrm{S} 1}$ plane-wave program, CASTEP, ${ }^{\mathrm{S} 2}$ with a single point in the Brillouin zone, and used the generalized gradient approximation (GGA) of Perdew and Wang (GGA-PW91). ${ }^{\mathrm{S}} \mathrm{A}$ kinetic energy cutoff of $280 \mathrm{eV}$ was used along with periodically repeating supercells. The distance between two nearest neighboring tubes was over $10 \AA$ to insure negligible 
interaction between the tube and its periodic images.

As shown in Fig.S2a, the optimal C1-C1 bond length of the 7-7 ring fusion in the defect tube is $1.35 \AA$, much shorter than the optimal C-C bond length $(\sim 1.42 \AA)$ in the perfect tube. The pyramidal angle at a $C$ atom of the perfect tube is $\sim 6.1^{\circ}$. The pyramidal angle at the $\mathrm{C} 1$ atom of the Stone-Wales defect is $\sim-2^{\circ}$, indicating inverse curvature at this site. This suggests that an incoming (especially a small) molecule would favor endo-mode access over exo-mode addition. Note that the pyramidal angle at the carbon atom of the peripheral 5-6-7 ring fusion around the Stone-Wales defect site (i.e. C2 atom in Fig. S2a) is $\sim 8.8^{\circ}, 2.7^{\circ}$ larger than that at a perfect site. Thus the C2 site could be more reactive in the defect tube.

The exothermicity of $\mathrm{O}_{3}$ addition to the $\mathrm{C} 1-\mathrm{C} 2$ pair site of the perfect tube (Fig.S1b) is computed to be $19.6 \mathrm{kcal} / \mathrm{mol}$, in good agreement with the B3LYP/6-31G* 13-layered cluster model (20.2 kcal/mol).

Four modes have been considered for the $\mathrm{O}_{3}$ addition: to the Stone-Wales defect site (the 7-7 ring fusion) in both exo- (Fig. S2b) and endo (Fig. S2e) modes; to two peripheral sites in exo-mode, i.e., the 5-6 ring fusion (Fig. S2c) and the 6-7 ring fusion (Fig. S2d), respectively. The following conclusions can be drawn from the computed reaction energies (Table $\mathrm{S} 1)$ :

(1) The exo-mode addition to the 7-7 ring fusion (Fig. S2b) is endothermic, whereas the endo-mode addition is exothermic, but only by $12.8 \mathrm{kcal} / \mathrm{mol}$. Thus, the central 7-7 ring fusion of the Stone-Wales defect should be less reactive than a perfect site.

(2) The additions to the peripheral 5-6 and 5-7 ring fusions (Fig. S2c and S2d, respectively) are much more exothermic, 49.3 and $29.8 \mathrm{kcal} / \mathrm{mol}$, respectively. The much higher exothermicity at these sites can be ascribed to the larger local strain at the 5-6-7 ring fusion apex (i.e. the $\mathrm{C} 2$ atom in Fig.S2a), where the local curvature is quite large $\left(\sim 8.8^{\circ}\right.$ pyramidal angle $)$.

This study shows how the different local curvature introduced by the topological defect might affect the chemical reactivity of the sites in its vicinity. 
Table S1 Reaction energies (RE, $\mathrm{kcal} / \mathrm{mol}$ ) for the addition of ozone to the perfect site and Stone-Wales defect sites in armchair $(5,5)$ single-wall carbon nanotube sidewall.

\begin{tabular}{lccccc}
\hline & Perfect tube & \multicolumn{4}{c}{ SW-defect tube } \\
\cline { 3 - 6 } Site $^{\mathrm{a}}$ & C1-C2 & C1-C1 (exo) & C1-C1(endo) & C2-C3 & C2-C4 \\
\hline RE & -19.6 & +11.6 & -12.8 & -49.3 & -29.8 \\
\hline
\end{tabular}

${ }^{\mathrm{a}}$ Refer to Fig.S1a and Fig. S2a for the definition of the pair site.

${ }^{\mathrm{b}} \mathrm{RE}=\mathrm{E}\left(\right.$ tube $\left.+\mathrm{O}_{3}\right)-\mathrm{E}($ tube $)-\mathrm{E}\left(\mathrm{O}_{3}\right)$. Negative $\mathrm{RE}$ means the addition is exothermic.

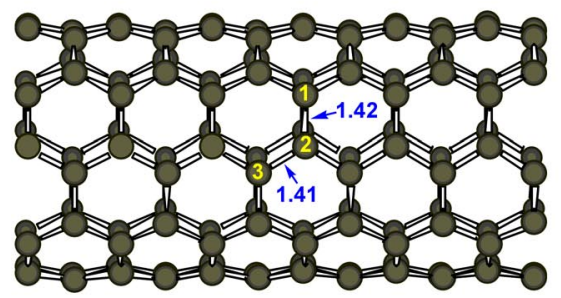

a)

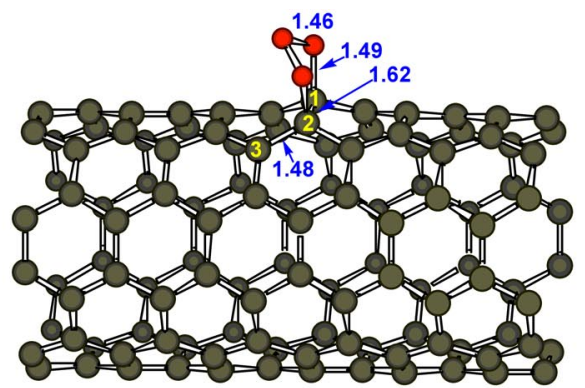

b)

Figure S1 a) a 120-atom supercell for a defect-free $(5,5)$ tube; b) optimized geometry for the addition of $\mathrm{O}_{3}$ to the $\mathrm{C} 1-\mathrm{C} 2$ pair site of a defect-free $(5,5)$ tube. (Bond lengths in A) 


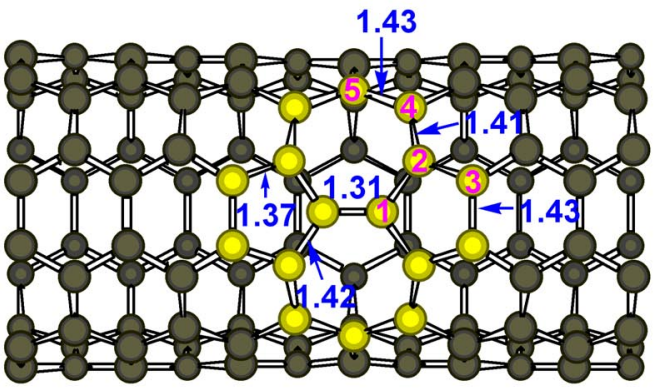

a)

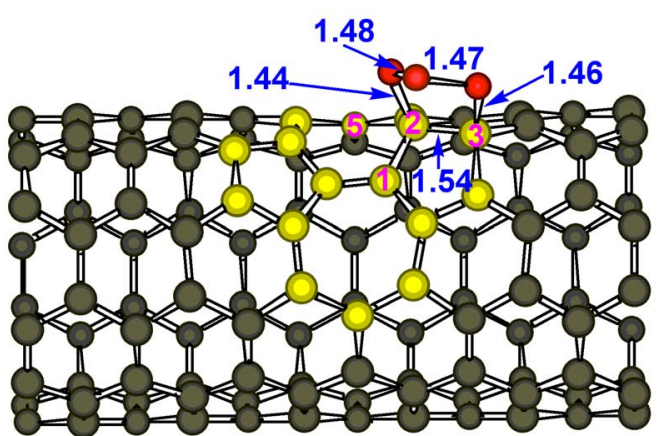

c)

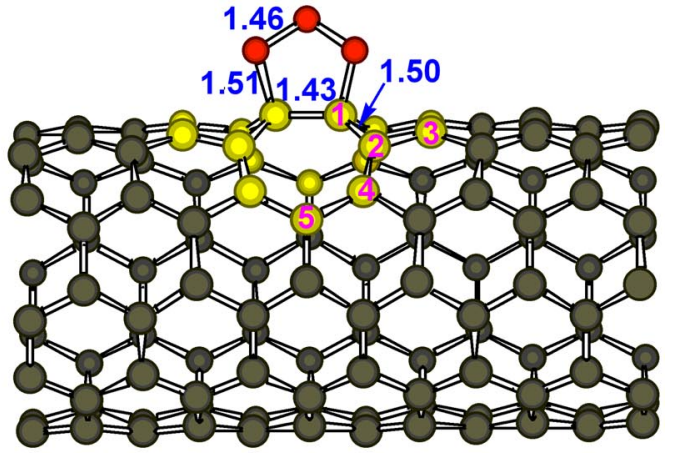

b)

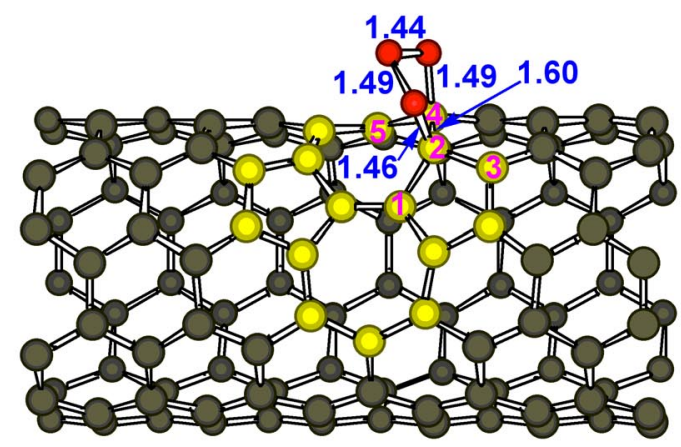

d)

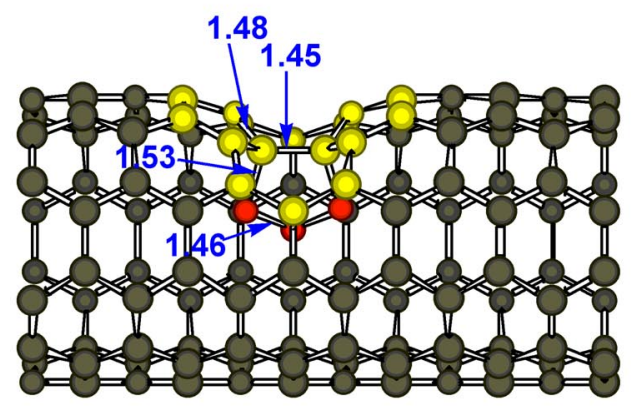

e)

Figure S2 Optimized geometries for a) the 120-atom supercell of a $(5,5)$ tube with a Stone-Wales defect (highlighted part), and for the products of $\mathrm{O}_{3}$ addition to $b$ ) the $\mathrm{C} 1-\mathrm{C} 1$ pair site (exo-mode), c) C2-C3 pair site, d) C2-C4 pair site, and e) C1-C1 pair site (endo-mode) of the defect $(5,5)$ tube. (Bond lengths in $\AA$ )

\section{References}

[S1] Vanderbilt, D. Phys. Rev. B 1990, 41, 7892.

[S2] Milman, V.; Winkler, B.; White, J. A.; Pickard, C. J.; Payne, M.C.; Akhmatskaya, E.V.; Nobes, R. H. Int. J. Quantum Chem. 2000, 77, 895.

[S3] Perdew, J. P.; Burke K.; Wang, Y. Phys. Rev. B 1996, 54, 16533. 